\title{
Correlaç̃o entre 0 índice de massa corporal e a Prega CuTÂNeA tricipital em criancas da cidade de Paulínia, São Paulo, SP
}

\author{
Mariana Porto Zambon; Maria de lurdes Zanolli; Denise Barbieri Marmo; Luís Alberto Magna; \\ luis Manuel Guimarey; André Moreno Morcillo \\ Trabalho realizado no Hospital das Clínicas da Unicamp - \\ Departamento de Pediatria, Campinas, São Paulo, SP.
}

RESUMO - 0 índice de massa corporal (IMC) tem sido indicado como método de escolha para o diagnóstico da obesidade em adultos e crianças.

OBJEtivo. Estudar a correlação existente entre o IMC e a prega cutânea tricipital (PCT).

Ме́тоDos. Foram avaliadas 4.236 crianças de 3,I a 10,9 anos, $48,3 \%$ do sexo masculino e $51,6 \%$ feminino, de quatro estudos realizados em Paulínia, SP, Brasil. Mediu-se o peso e a estatura, com os quais calculouse o IMC. A PCT foi medida com paquímetro Holtain. Os dados foram transformados em escores $\mathbf{Z}$ e comparados à curva norte-americana (Frisancho, 1993). A população foi dividida em grupos de acordo com os escores $Z$ do IMC: grupo A) escore $Z \leq-$ I; B) - I < escore $Z<$ I; C) escore $Z \geq I$. Realizou-se uma análise de regressão linear múltipla (método stepwise). Os dados foram processados no SPSS.
Resultados. 0 grupo $A$ apresentou menor variabilidade na PCT ( $\bar{z}$ 7,8; $D P=2,3)$ quando comparado aos demais $(B: \bar{X}=$ 10,I; $D P=4$ e $C: \bar{X}=17,8 ; D P=4,4) . \quad N a$ análise da regressão linear, considerando todos os casos, a PCT apresentou $R^{2}=0,478$. Nos grupos B e C a PCT teve $R^{2}=0,364$ e 0,368 , respectivamente enquanto no grupo $A$ foi apenas 0,032 .

ConcLusão. A correlação entre o IMC e a PCT é elevada no grupo de crianças com risco de obesidade (grupo C), o que justifica a substituição do uso da PCT pelo IMC em estudos populacionais de obesidade em escolares brasileiros.

UnItermos: Avaliação nutricional. Índice de massa corporal. Prega cutânea tricipital.

\section{INTRODUÇÃo}

Atualmente considerada um problema de saúde pública, a obesidade é definida como um acúmulo excessivo de gordura provocado por um balanço positivo de energia ${ }^{1,2}$. Seus estudos de prevalência e incidência na infância e na adolescência apresentam dificuldades de realização pelas diferenças de definição, pela escolha do método diagnóstico e pelo referencial utilizado ${ }^{2,3}$.

A medida das dobras cutâneas vem sendo utilizada, em estudos populacionais, para a avaliação do tecido adiposo subcutâneo, principalmente paraidentificar os pacientes com excesso de gordura. Porém, tem como desvantagem a dificuldade técnica de padronização ${ }^{4}$.

Várias pregas cutâneas podem ser avaliadas isoladamente ou em conjunto. Entre estas encontramos a prega cutânea bicipital (PCB), a tricipital (PCT), a subescapular (PCSE) e a suprailíaca (PCSI). Em crianças a PCT é a mais utilizada 5 .

* Correspondência:

R: Botafogo 151/491 Caminhos de San Conrado - Sousas

Campinas - SP - CEP: 13.130-601 Fax: (55-19) $3788-8260$

Email:zambon@correionet.com.br
Oíndice de massa corporal (peso/altura²) tem sido preconizado como um bom método para avaliação da obesidade, tanto em crianças como em adolescentes e adultos ${ }^{6,7,8}$.

O objetivo deste trabalho foi estudar a correlação existente entre o índice de massa corporal (IMC) e a prega cutânea tricipital (PCT) em crianças.

\section{Métodos}

Foram incluídas 4.236 crianças [48,3\%M;5I,6\%F]comidades entre 3, I a I0,9 anos $(7,9 \pm 1,4)$. Estegrupocorrespondea estudantes de escolas públicas de quatro coortes realizadas na cidade de Paulínia, São Paulo, Brasi $\left.\right|^{9,10,11,12}$. Da primeira, realizada entre setembro de 1979 e novembro de 1980 com 625 escolares, foram incluídas 620 crianças, 357 do sexo masculinoe 263 do feminino?. Do segundo estudo, realizado entre março de 1984 e novembro de 1985 que incluiu 1605 estudantes do primeiro grau, analisamos 1509:732 do sexo masculino e 858 do feminino ${ }^{10}$. Um terceiro estudo avaliou 52 I crianças de um total de 229 I matriculadas em pré-escolas, no período de julho a novembro de 1990, as quais foram selecionadas por amostragem aleatória estratificada. Destas, estudamos 518 , sendo 260 do sexo masculino e 258 do feminino". Em outro estudo realizado entre março de 1993 e novembro de 1994 com 1903 escolares de primeiro grau, incluímos 1508, sendo 837 do sexo masculino e $67 /$ do feminino ${ }^{12}$.

As crianças foram submetidas a um exame antropométrico, sendo queem todos os estudos foram medidos o peso, a altura e a PCT empregando a mesma técnica ${ }^{13}$. A altura foi medida com um antropômetro de madeira (precisão $0,1 \mathrm{~cm})$, o peso em uma balança mecânica (precisão l00g) ea prega cutânea tricipital com um paquímetro Holtain com escala de $0,2 \mathrm{~mm}$. No cálculo dos escores $Z$ do IMC, utilizou-se como referencial a curva norte-americana ${ }^{14}$.

Para se estudar a relação existente entre 0 IMC e a PCT empregou-se a regressão linear múltipla (método stepwise), considerando como variáveis independentes a idade, o sexo e a prega cutânea tricipital ${ }^{15}$. Procurou-se um modelo que se ajustasse a toda população, a qual, posteriormente, foi dividida em três grupos de acordo com o IMC: Grupo (A) escore $Z \leq-I$; Grupo (B) - I < escore $Z<$ I e grupo $(C)$ escore $Z \geq 1$. Os dados foram processados empregando o software SPSS. 
Tabela - I Características quanto à idade (anos) e sexo em relação as quatro coortes

\begin{tabular}{|c|c|c|c|c|c|c|c|c|c|c|c|c|}
\hline \multirow{3}{*}{ Sexo } & \multicolumn{12}{|c|}{ COORTES } \\
\hline & \multicolumn{3}{|c|}{ Guimarey(1983) } & \multicolumn{3}{|c|}{ Morcillo (1987) } & \multicolumn{3}{|c|}{ Zanolli (1992) } & \multicolumn{3}{|c|}{ Marmo(1999) } \\
\hline & $\mathrm{n}$ & $\bar{\chi}$ & {$[\min -\max ]$} & $n$ & $\bar{\chi}$ & [min-max] & $n$ & $\bar{\chi}$ & [min-max] & $n$ & $\bar{\chi}$ & [min-max] \\
\hline Masculino & 263 & 7,9 & {$[3,2-9,9]$} & 858 & 7,9 & {$[6,3-9,9]$} & 258 & 5,7 & {$[3,3-7,6]$} & 671 & 8,1 & {$[5,9-9,9]$} \\
\hline Feminino & 357 & 8,4 & {$[3,1-10,9]$} & 732 & 8,4 & {$[6,4-10,9]$} & 260 & 5,7 & {$[3,2-7,4]$} & 837 & 8,5 & {$[5,6-10,9]$} \\
\hline Total & 620 & 8,2 & {$[3, \mid-10,9]$} & 1590 & 8,1 & {$[6,3-10,9]$} & 518 & 5,7 & {$[3,2-7,6]$} & 1508 & 8,3 & {$[5,6-10,9]$} \\
\hline
\end{tabular}

Tabela - 2 Média, desvio padrão e coeficiente de variação da prega cutânea tricipital em relação aos grupos de IMC

\begin{tabular}{lccc}
\hline \multicolumn{1}{c}{ IMC } & \multicolumn{3}{c}{ PCT } \\
\cline { 2 - 4 } & $\mathbf{N}$ & Média(DP)* & CV** \\
(A) Escore $Z \leq-1,0$ & 1.176 & $7,8(2,3)$ & 29,4 \\
(B) $-1,0<$ escores $Z<1,0$ & $2.79 \mid$ & $10,1(4,0)$ & 39,6 \\
(C) Escore $Z \geq 1$ & 269 & $17,8(6,2)$ & 34,8 \\
TODOS & 4.236 & $9,9(4,4)$ & 44,4 \\
\hline
\end{tabular}

*Média (desvio padrão) $=m m$

***Coeficiente de variação(\%)

Tabela - 3 Regressão linear múltipla (modelos finais) do IMC em relação à idade, sexo e PCT

\begin{tabular}{|c|c|c|}
\hline IMC & R2* & Regressão Linear Múltipla*** \\
\hline (A) Escore $Z \leq-1,0$ & $0,254^{\#}$ & $y=0, \mid 77$ Idade $+0,26 \mid$ Sexo $+0,056$ PCT $+1 \mid, 923$ \\
\hline (B) $-1,0<$ escore $Z<1,0$ & $0,\left.44\right|^{\# \#}$ & $y=0,159 \mid$ dade $+0,383$ Sexo $+0,162$ PCT $+12,848$ \\
\hline (C) Escore Z $\geq 1$ & $0,44 !$ & $y=0,393$ Idade $+0,1 \mid 4 P C T+14,846$ \\
\hline TODOSOSCASOS & $0,506 !$ & $Y=0,287$ PCT $-0,606$ SEXO + 12,457 \\
\hline \multicolumn{2}{|c|}{ 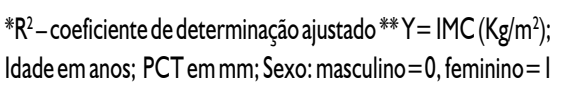 } & $\begin{array}{l}\left.\# \#-R^{2} \text { (idade }\right)=0,050 \quad R^{2}(\text { sexo })=0,027 \quad R^{2}(P C T)=0,364 \\
\left.! R^{2} \text { (idade }\right)=0,073 \quad R^{2}(P C T)=0,368\end{array}$ \\
\hline$\#-R^{2}($ idade $)=0,203 \quad R^{2}($ sexo $)$ & $R^{2}(P C T)=0,032$ & $! ! R^{2}(\operatorname{sexo})=0,028 \quad R^{2}(P C T)=0,478$ \\
\hline
\end{tabular}

\section{Resultados}

A distribuição das crianças em relação ao sexo, idade e coorte de origem é apresentada na Tabela I.

$\mathrm{Na}$ Tabela 2 são apresentadas a média, o desvio padrão e o coeficiente de variação da $P C T$ em relação aos grupos $A, B$ e $C$ de IMC. $O$ grupo $A$, constituído por crianças que têm escores $Z$ de IMC menor ou igual a $-1,0$, tem menor variabilidade na PCT quando comparado aos demais.
0,364 e 0,368 , respectivamente. No grupo B, a idade apresenta $R^{2}$ igual a 0,050 e o sexo $R^{2}$ igual a 0,027 , enquanto no grupo $C$ a idade determina um aumento $R^{2}$ de 0,073 . Os modelos finais são apresentados na Tabela 3.

\section{Discussão}

O IMC tem sido recomendado para classificar o sobrepeso e a obesidade em adultos ${ }^{2}$. É muito útil para estimar a prevalência da obesidade em populações, no entanto pouco contribui para se estudar a distribuição do tecido adiposo, pois valores altos do IMC não estão necessariamente associados a uma maior quantidade de tecido adiposo. Além disso, pode não haver correspondência em relação ao risco de morbidade entre diferentes indivíduos com semelhantes valores de IMC ${ }^{2}$.

Estudos realizados em adultos têm demonstrado que há uma correlação positiva entre o IMC e a gordura corporal, seja ela expressa pela porcentagem de gordura $(\% \mathrm{G})$ ou gordura corporal total (GCT) $)^{16,17}$. No entanto, Revick \& Israel $(1986)^{18}$ e Roubenoff et al. $(1995)^{19}$ afirmam que o seu uso deve ser cuidadoso, pois obtiveram estimativas imprecisas da GCT e da \%G.

No que se refere às crianças e adolescentes, são inúmeras as preocupações com relação ao uso do IMC: a escolha da curva de referência, os pontos de corte, a interferência do sexo, da idade e da etnia $a^{4,7,20,21,22}$.

Quanto aos estudos de validação neste grupo etário, Pietrobelli et al. $(1998)^{23}$, avaliando a composição corporal por Dual Energy X-Ray Absorptiometry (DEXA) em crianças e adolescentes (5-19 anos), demonstraram uma forte associação entre 0 IMC e a GCT $\left(R^{2}=0,85\right.$ e $R^{2}=0,89$, respec- 
tivamente, para os sexos masculino e feminino) e entre o IMC e a \%G $\left(R^{2}=0,63\right.$ e $R^{2}=0,69$, respectivamente, para $\circ$ sexo masculino e feminino). Dietz \& Bellizzi $(1999)^{7}$, revendo trabalhos de validação entre o IMC e a porcentagem de gordura, referem coeficientes de correlação que variam de 0,50 a 0,87 nos estudos que utilizaram a DEXA e de 0,44 a 0,77 para a hidrodensitometria.

Malina \& Katzmarzyk (1999) ${ }^{8}$, analisando os dados de seis estudos com crianças e adolescentes de 9 a 19 anos, calcularam a sensibilidade e a especificidade do IMC no diagnóstico do sobrepeso e da obesidade. Quando comparado com os resultados obtidos no diagnóstico do sobrepeso por hidrodensitometria, a sensibilidade (4,3\%-30,8\%) era muito menor que a especificidade (95\%-98,8\%). Quando comparado com a PCT, a sensibilidade (20\%-75\% para o sobrepeso e I4,3\%-60\% para a obesidade) também era menor que a especificidade (86,1\%-98,1\% para 0 sobrepeso e $96,3 \%$ - $100 \%$ para a obesidade). Estes achados são relevantes, já que indicam que o IMC pode não ser um bom método para avaliação da obesidade, corroborando as preocupações de Revick \& Israel $(1986)^{18} \mathrm{e}$ Roubenoff et al. (1995) ${ }^{19}$.

O método alternativo para ser usado em estudos populacionais é a medida das dobras cutâneas, que é uma medida direta da espessura do tecido adiposo do celular subcutâneo, no entanto, a medida das dobras cutâneas apresenta dificuldades com relação ao processo de treinamento, precisão e reprodutibilidade dos resultados.

Ward et al. (1975) ${ }^{24}$ demonstraram quea correlação entre a PCT e a GCT $\left(D_{2} O\right)$ em adultos era respectivamente 0,56 e 0,87 para homens e mulheres, enquanto para a prega cutânea subescapular (PCSE) era de 0,72 (mulheres) e 0,54 (homens). Goran \& Gower $(1999)^{25}$ afirmam que a correlação entre a gordura visceral e as dobras cutâneas é bastante alta $(r=0,85$ para PCSE, $r=0,92$ para a $\mathrm{PCT}, r=0,91$ para a prega cutânea abdominal e $r=0,91$ para a prega cutânea supra-ilíaca), porém Schoroeder \& Martorell $(1999)^{26}$ encontraram pobre correlação entre o IMC e os indicadores de obesidade central.

Bolzan et al. (1999)27, estudando 321 crianças (6-13 anos) de General Lavalle - Argentina, encontraram um coeficiente de cor- relação entre o PCT e o IMC de 0,76. No Brasil, Fonseca, etal. (1998) ${ }^{28}$, estudando 39I adolescentes (15-17 anos) da cidade de Niterói, Rio de Janeiro, encontraram correlação bastante alta entre a PCT e o IMC $(0,73$ e $0,80)$, respectivamente no sexo masculino e feminino) e PCSE e IMC $(0,78$ e 0,80$)$, respectivamente no sexo masculino e feminino). Gamba $(1999)^{29}$ também encontrou elevada correlação entre a PCT e o IMC, com valores de $\boldsymbol{r}$ variando de 0,66 a 0,75 no sexo masculino e de 0,63 a $0,8 \mathrm{I}$ no feminino.

Considerando toda a população, nossos resultados demonstram que a $\mathrm{PCT}$ foi a principal responsável pela variação do IMC sendo pequena, porém significativa a contribuição da idade e do sexo. Ao separarmos a população em três grupos, observamos que para o grupo $A(I M C Z \leq-I)$ o modelo de regressão apresenta o menor coeficiente de correlação e que a PCT deixa de ser a variável que mais contribui para a variação do IMC. No entanto, no grupo C (IMC Z ${ }^{3}$ I) a PCT tem contribuição significativa e maior que a idade. Pietrobelli et al. (1998) também concluem que valores altos de IMC estão associados com maior adiposidade.

O fato do modelo explicar pouco mais de $50 \%$ da variação do IMC, justifica-se por não terem sido consideradas a gordura visceral, a massa muscular e a massa óssea. Levando-se em conta a sua simplicidade, o baixo custo e a alta reprodutibilidade, recomendamos a utilização do IMC em estudos de prevalência de obesidade em pré-escolares e escolares.

\section{Conclusão}

Estes resultados sugerem uma associação entre o IMC e a PCT, que é uma medida direta da adiposidade.

SUMMARY
BODY MASS INDEX AND TRICEPS
SKINFOLD CORRELATION IN CHILDREN FROM
PAULínIA CITY, SÃO PAULO, SP
Bodymassindex (BMI) has beenconsidered
acriteriontodefineandanalyseobesityinadults
and children.
BACKGROUND. thepurposeofthisstudywasto
evaluate the correlation between BMI and
tricepsskinfold(TSF).
MeTHODS. therewerestudied4,236children
(3.I-I0.9y); 48.3\%M:5I.6\%F, from four

studies made in Paulínia, SP-Brazil. Height, weightand TSF(Holtain caliper)weremeasured. For each children BMI was calculated and transformed in SDS, according to North American data (Frisancho, 1993). Multiple linear regression analysis (stepwise) was used for the whole population and in three groups according to BMI:A)SDS $\leq-1.0 ; B)-1.0<S D S<1.0 ; C)$ $S D S{ }^{3}$ I.O. Data were processed with SPSS software.

RESULTS. in group A, the TSF $(7.8 \pm 2.3)$ variability was lower when compared with the groups $B(I 0 . I \pm 4.0)$ and $C(I 7.8 \pm 6.2)$. In multiple linear regression with the whole population, $R^{2}=0.478$ for TSF. In groups $B$ and $C, R^{2}=0.364$ and 0.368 respectively for TSF, and in group $A$ it was only 0.032 .

CONCLUSIONS. these observations demonstrateda height correlation between BMland TSF inchildren withobesity risk (group C). Therefore, we conclude that, in Brazil BMI can be used for children's research of obesity in population studies, instead of TSF. [Rev Assoc Med Bras 2003; 49(2): 137-40]

KEY WORDS: Nutritional assesment. Body mass index. Triceps skin fold.

\section{REFERÊNCIAS}

I. Millar WJ, Stephens,T. The prevalence of overweight and obesity in Britain, Canada and United States. Am J Public Health 1987; 77:38-4I.

2. WHO. World Health Organization. Obesitypreventing and managing the global epidemic. Geneva: WHO; 1997.

3. Marmo DB. Análise comparativa de cinco parâmetros antropométricos para o diagnóstico de obesidade em escolares da cidade de Paulínia-São Paulo (dissertação). Campinas: Faculdade de Ciências Médicas, Universidade Estadual de Campinas-Unicamp; 1988.

4. Bellizzi MC, Dietz WH. Workshop on childhood obesity: summary of the discussion Am J Clin Nutr 1999; 70: 173-5S.

5. Mascarenhas MR, Zemel B, Stallings VA. Nutritional assessment in pediatrics. Nutrition 1998; 14:105-15.

6. Rolland-Cachera MF, Sempé M, GuilloudBataille M, Patois E, Pequignot-Guggenbuhl F et al. Adiposity indices in children. Am J Clin Nutr 1982; 34: I 78-84.

7. Dietz WH, Bellizzi MC. Introduction: the use of body mass index to assess obesity in children. Am J Clin Nutr 1999; 70: 1 23-5S.

8. Malina RM, Katzmarzyk PT. Validity of the body mass index as na indicator of the risk and presence of overweight in adolescents. Am J Clin Nutr 1999; 70: 1 31-6S. 
9. Guimarey LM. Crescimento e estado nutricional em escolares de Paulínia, São PauloBrasil (tese). Campinas: Faculdade de Ciências Médicas, Universidade Estadual de CampinasUnicamp; 1983.

10. Morcillo AM. Estudo comparativo de sete parâmetros antropométricos em escolares da cidade de Paulínia - São Paulo, referente aos períodos de 1979// 980 e 1984/1 985 (tese). Campinas: Faculdade de Ciências Médicas, Universidade Estadual de Campinas-Unicamp; 1987.

II. Zanolli ML. Avaliação nutricional de préescolares matriculados nas escolas municipais de educação infantil de Paulínia - SP (dissertação). Campinas: Faculdade de Ciências Médicas, Universidade Estadual de CampinasÚnicamp; 1987.

12. Marmo DB. Avaliação da tendência secular da altura, do peso e da relação peso-altura de uma população de escolares da cidade de Paulínia São Paulo. Comparação entre os períodos 1979/1980 e 1993/1994 (tese). Campinas: Faculdade de Ciências Médicas, Universidade Estadual de Campinas-Unicamp; 1999.

13. Cameron N. The methods of auxological anthropometry. In: Falkner F, Tanner J M., editors. Human growth. New York: Plenum Press; 1978. p.35-90.

14. Frisancho AR. Anthropometric standards for the assessment of growth and nutritional status. Michigan: The University of Michigan Press; 1993.
15. Zar JH. Biostatistical analysis. New Jersey: Prentice-Hall Inc.; 1984. p.328-33.

16. Gallagher D, Visser M, Sepúlveda D, Pierson RN, Harris T, Heymsfield SB et al. How useful is body mass index for comparison of body fatness across age, sex, and ethnic groups? Am JEpidemiol 1996; I 43:228-39.

17. Morabia A, Ross A, Curtin F, Pichard C, Slosman DO. Relation of BMI to a dual-energy $\mathrm{X}$-ray absortiometry measure of fatness. $\mathrm{Br}$ J Nutr 1999; 82:49-55.

18. Revicki DA, Israel RG. Relationship between body mass indices and measures of body adiposity. AmJ Public Health 1986; 76:992-4.

19. Roubenoff R, Dallal G, Wilson P. Predicting body fatness: the body mass index vs estimation by bioelectrical impedance. Am J Public Health 1995; 85:726-8.

20. Daniels SR, Khoury PR, Morrison JA.. The utility of body mass index as a measure of body fatness in children and adolescents: differences by race and gender. Pediatrics 1997; 99:804-7.

21. Dietz WH, Robinson TN. Use of the body mass index (BMI)as ameasure of overweight in children and adolescents. J Pediatr 1998; | 32: I9|-3.

22. Guillaume M. Defining obesity in childhood: current practice. Am J Clin Nutr 1999; 70:126-30S.

23. Pietrobelli A, Faith MS, Allison DB, Gallagher D, Chuimell G, Heymsfield SB et al. Body mass index as a measure of adiposity among children and adolescents: a validation study. J Pediatr 1998; 1 32:204-10.
24. Ward GM, Krzywicki HJ, Rahman DP, Quaas RL, Nelson RA, Consolazio CF et al. Relationship of anthropometric measurements to body fat as determined by densitometry, potassium-40, and body water. Am J Clin Nutr 1975; 28:162-9.

25. Goran MI, Gower BA. Relation between visceral fat and disease risk in children and adolescents. Am J Clin Nutr 1999; 70:149$56 \mathrm{~S}$.

26. Schroeder DG, Martorell R. Fatness and body mass index from birth to young adulthood in a rural Guatemalan population. Am J Clin Nutr 1999; 70:137-44S.

27. Bolzan A, Guimarey L, Frisancho AR. Study of growth in rural school children from Buenos Aires, Argentina using upper arm muscle area by height and other anthropometric dimensions of body composition. Ann Hum Biol 1999; 26: 185-93.

28. Fonseca VM, Sichieri R, Veiga GV. Factors associated with obesity among adolescents. J Public Health 1998; 32:54 I-9.

29. Gamba ME. Avaliação do índice de massa corporal em escolares de Campinas - São Paulo (dissertação). Campinas: Faculdade de Ciências Médicas, Universidade Estadual de Campinas-Unicamp; 1999.

Artigo recebido:01/II/200I

Aceito para publicação: 06/12/2002 